\title{
In vitro effect of bergamot (Citrus bergamia) juice against cagA-positive and-negative clinical isolates of Helicobacter pylori
}

\author{
Angela Filocamo ${ }^{1}$, Carlo Bisignano ${ }^{2}$, Nadia Ferlazzo ${ }^{1}$, Santa Cirmi ${ }^{1}$, Giuseppina Mandalari ${ }^{1 *}$ and Michele Navarra ${ }^{1 *}$
}

\begin{abstract}
Background: Helicobacter pylori infection has been associated with chronic gastritis, peptic ulcer and gastric carcinoma as over half of the world's population is colonized with this gram-negative bacterium. Due to the increasing antibiotic resistance, its eradication rates fails in a great portion of patients. A number of studies showed that molecules largely distributed in commonly consumed fruits and vegetables may have antimicrobial activity. The aim of the present study was to investigate the effect of bergamot juice (BJ) against Helicobacter pylori in vitro. The potential therapeutic combination between BJ and the antibiotics amoxicillin (AMX), clarithromycin (CLA) and metronidazole (MTZ) has also been evaluated.
\end{abstract}

Methods: The minimum inhibitory concentration (MIC) of BJ, AMX, CLA and MTZ against 2 ATCC and 32 clinical isolates of $\mathrm{H}$. pylori was assayed according to CLSI. The checkerboard method was used to determine the efficacy of the association BJ with the three reference antibiotics.

Killing curves were performed on the two cagA-positive ATCC strains of H. pylori (ATCC 43504 and ATCC 49503), on the clinical isolate cagA-positive HP6 strain of H. pylori and on the clinical isolate cagA-negative HP61 strain of H. pylori.

Results: BJ $(2.5 \%, \mathrm{v} / \mathrm{v})$ inhibited the growth of $50 \%$ of the $\mathrm{H}$. pylori clinical isolates, whereas $5 \%(\mathrm{v} / \mathrm{v})$ inhibited $90 \%$. AMX was the most effective antibiotic against the reference strains and the clinical isolates, followed by CLA and MTZ. In the combination assays, synergism was observed between BJ and AMX and between BJ and MTZ against both the reference strains and the clinical isolates. Indifference was observed between BJ and CLA.

Conclusions: BJ was effective in vitro against $H$. pylori and the genotype status of the clinical strains may have an impact on its susceptibility. The synergistic combination of BJ and antibiotics could be used to prevent or treat resistance.

Keywords: Bergamot, H. pylori, Antimicrobial, Citrus bergamia, Natural products, Antibiotic resistance, Synergism, Complementary and alternative medicines

\section{Background}

Infection with Helicobacter pylori (H. pylori), identified as group 1 carcinogen by the International Agency for Research on Cancer, has been associated with chronic gastritis, peptic ulcer and gastric carcinoma [1, 2]. Its incidence is highly variable in relation to geography, ethnicity, age and socioeconomic factors. Infection rates are similar for men and women and increase progressively with age. The

\footnotetext{
*Correspondence: gmandalari@unime.it; mnavarra@unime.it

'Dipartimento di Scienze del Farmaco e Prodotti per la Salute, University of Messina, Viale Annunziata, 98168, Messina, Italy

Full list of author information is available at the end of the article
}

estimated prevalence is $70 \%$ in developing countries, mainly in the young population, and $30 \%-40 \%$ in the developed countries [3].

The virulence of the infecting strain is believed to affect the severity of gastroduodenal diseases caused by $\mathrm{H}$. pylori. Despite the wide genetic diversity of $H$. pylori involved in its pathogenesis, a number of genetic loci, such as the cytotoxin-associated gene ( $\operatorname{cag} \mathrm{A})$ and the vacuolating cytotoxin gene $(v a c \mathrm{~A})$, have been identified. VacA, present in all $H$. pylori strains, contains two variable parts relevant to virulence [4], whereas cagA, not present in every H. pylori strain, is a marker for a pathogenicity island (PAI) [5] 
associated with more severe clinical outcomes and an increased risk of developing gastric cancer and peptic ulcer [6]. Although heterogeneity in single cagA and $v a c \mathrm{~A}$ genes in $H$. pylori have been associated to specific clinical effects [7], some studies suggested that genetic heterogeneity is due to geographical diversity and host environment [8].

The standard treatment for $H$. pylori related disease is a combination of antimicrobial agents and anti-acid agents [9]. The first line of treatment regimens consist of a triple therapy, carried out through the recruitment of a proton pump inhibitor (PPI) and two antibiotics, amoxicillin (AMX) and clarithromycin (CLA), or metronidazole (MTZ) and CLA. Quadruple therapy obtained by the association of PPI, bismuth and two antibiotics (AMX + CLA, or MTZ + tetracycline (TET)) is equivalent or little superior in the eradication rates, although reducing the compliance [3]. However, standard PPIbased therapy fails in up to $30 \%$ of patients, and eradication rates were reduced to $70-80 \%$ over the last few years. This is mostly due to increasing antibiotic resistance which favors recurrence of $H$. pylori infection, particularly high in developing countries.

Due to common side effects and the development of antimicrobial resistance [10], a number of natural compounds have been tested as alternatives [11-13]. We have recently demonstrated that polyphenolic fractions from almond skin were active against standard strains and clinical isolates of $H$. pylori [14].

Citrus bergamia Risso et Poiteau (bergamot) is an endemic plant of the Calabrian region in Southern Italy, mostly used for the extraction of its essential oil from the fruit peel, much employed in the fragrance industry and lesser exploited in pharmaceutical and food applications. Bergamot juice (BJ), obtained from the endocarp of the fruit, is considered a byproduct barely utilized by the food industry [15]. Recently, we focused on potential beneficial effects of BJ. We demonstrated that BJ inhibited signaling pathways linked to cancer-associated aggressive phenotype, thus reducing proliferation, adhesion and migration both in vitro [16] and in vivo [17]. In addition, we documented that the antiproliferative effect of BJ was due to its flavonoid fraction (BJe), which inhibited proliferation inducing apoptosis in human colon cancer cells [18]. Moreover, we provided evidence that BJe possess antioxidant effects [19] and inhibited both gene expression and secretion of LPS-induced proinflammatory cytokines in a model of LPS-stimulated THP-1 cell line [20]. Moreover, BJe exerted antiinflammatory effects also in an animal model of experimental colitis [21], suggesting a possible role in treating rheumatoid arthritis with a good balance between efficacy and safety [22]. Earlier studies demonstrated that the polyphenolic fraction of BJ reduced serum cholesterol, triglycerides and glycaemia in patients suffering from metabolic syndrome [23], supporting previous findings obtained in animal models [24].

The aim of the present study was to investigate the antimicrobial properties of $\mathrm{BJ}$ against $H$. pylori strains and the interaction between $\mathrm{BJ}$ and the antibiotics AMX, CLA and MTZ.

\section{Methods}

\section{Bergamot juice}

BJ was obtained by hand-squeezing fruits of Citrus bergamia Risso et Poiteau collected in the Calabrian region (Southern Italy). Prof. Antonio Rapisarda (pharmaceutical botany expert, University of Messina) has provided the identification of Citrus bergamia fruits used to obtain the bergamot juice employed in this experimental study. A voucher specimen of the plant, identified according to the botanical literature, was deposited in the herbarium (H.N. 3263-3266 \# CBSD) of the Dipartimento di Scienze del Farmaco e Prodotti per la Salute of University of Messina (Italy).

Aliquots of BJ were stored at $-20{ }^{\circ} \mathrm{C}$ until further use. The flavonoid composition of $\mathrm{BJ}$ was evaluated by RP-HPLC as previously reported [16]. The major flavonoids identified in BJ were eriocitrin, neoeriocitrin, naringin and neohesperidin, of which the most abundant compound was neoeriocitrin (29\% of total), followed by neohesperidin ( $27 \%$ of total). No significant differences in amounts of eriocitrin and naringin were detected. Minor identified compounds included a number of glucosides, such as apigenin-6,8-di-C-glucoside, diosmetin-6,8-di-C-glucoside and crisoeriol-7-O-neohesperidoside-49-glucoside.

\section{Patients, H. pylori strains and culture conditions}

Two reference American Type Culture Collection strains of Helicobacter pylori (ATCC 43504 and ATCC 49503) and thirty two clinical isolates recovered from gastric biopsy samples of dyspeptic adults (23 women, 9 men; average age, 51 years) undergoing digestive endoscopy at the Endoscopy Unit of the Department of Internal Medicine of the University of Messina, Messina, Italy, were used in this study [14]. None of the patients had previously undergone eradication therapy. All study subjects gave their informed consent and the study was approved by the local ethical committee (Comitato Etico Scientifico A.O.U. Policlinico "G. Martino", Messina, Italy). Diagnosis of peptic ulcer and non-ulcer dyspepsia (NUD) or gastritis was based on endoscopic examination of the stomach and duodenum. Biopsy samples were taken for each patient for culture. Isolates were derived from patients suffering from gastritis $(n=27$; $84.37 \%)$, or NUD ( $n=5 ; 15.62 \%)$.

Gastric biopsy specimens were placed in the sterile screw-capped tubes containing $0.5 \mathrm{ml}$ sterile saline and transported to the microbiology laboratory within $2 \mathrm{~h}$. 
Samples were soaked and sowed in selective (Pylori agar, BioMérieux, Florence, Italy) and non-selective (Columbia agar with $7 \%$ horse blood, CB, Oxoid, Milan, Italy) culture media. Cultures were incubated for 7 days at $37{ }^{\circ} \mathrm{C}$ under microaerophilic conditions. Grown bacteria were identified as $H$. pylori by typical morphology, Gram staining results and positive reactions to oxidase, catalase, and urease activities. The molecular identification of the tested strains has been previously reported [14].

All strains were harvested by suspension in Brucella broth (Becton Dickinson Italia, Milan, Italy) supplemented with $10 \%$ fetal bovine serum (BB, Euroclone, Milan, Italy) and $30 \%$ glycerol and stored in liquid nitrogen until used.

\section{Susceptibility studies}

The minimum inhibitory concentration (MIC) of BJ, amoxicillin (AMX, Sigma, Milan, Italy), clarithromycin (CLA, Sigma, Milan, Italy) and metronidazole (MTZ, Sigma, Milan,Italy) was assayed by the standard agar dilution method according to the guidelines of the National Committee for Clinical Laboratory Standards [25] using CB. Frozen stock cultures were thawed and subcultured on $\mathrm{CB}$ and grown for 3 days under microaerophilic conditions. Bacterial growth was taken from the plates, resuspended in $\mathrm{BB}$ and grown under shaking (125 rpm) at $37^{\circ} \mathrm{C}$ for $24 \mathrm{~h}$. H. pylori cultures in the exponential phase of growth were diluted with $\mathrm{BB}$ to contain about $5 \times 10^{7} \mathrm{CFU} / \mathrm{ml}$ by adjusting the turbidity of the suspension to match the MacFarland no. 1 standard. Tenmicroliter aliquots of the suspensions were inoculated on $\mathrm{CB}$ containing twofold serial dilutions of the compound tested, ranging from $80(\%, \mathrm{v} / \mathrm{v})$ to $0.156(\%, \mathrm{v} / \mathrm{v})$. Compound-free CB media were included in each experiment to confirm the viability of the inoculum and to observe the growth of any contaminants. All plates were incubated at $37{ }^{\circ} \mathrm{C}$ in a microaerophilic atmosphere and examined after 3 days. The MIC was considered the lowest concentration at which the compound inhibited the development of visible bacterial growth on the agar plates.

\section{Combination assays}

In the combination assays, the "checkerboard" procedure described by White et al. [26] was followed to determine the efficacy of the association $\mathrm{BJ}$ with the three reference antibiotics against all tested strains. The test compounds represented by each antibiotic (AMX, CLA or MTZ) was serially diluted on the $\mathrm{x}$ axis, ranging from 1 to $1 / 64$ $x$ MIC, with increasing concentrations of $B J$ ranging from $1 / 32$ to $1 \times$ MIC on the $y$ axis.

MIC data were converted into fractional inhibitory concentration (FIC), defined as ratio of the concentration of the antimicrobial at an inhibitory concentration with a second compound to the concentration of the antimicrobial by itself as previously reported [27].

$\mathrm{FIC}_{\mathrm{A}}=\mathrm{MIC}$ of $\mathrm{A}$ with $\mathrm{B} / \mathrm{MIC}$ of $\mathrm{A}$

The FIC index was then calculated as follows: FIC index $=\mathrm{FIC}_{\mathrm{A}}+\mathrm{FIC}_{\mathrm{B}}$.

All MIC determinations were performed in duplicate for each strain.

\section{Time-kill curves}

Killing curves were performed on the two cagA-positive ATCC strains of $H$. pylori (ATCC 43504 and ATCC 49503), on the clinical isolate cagA-positive HP6 strain of $H$. pylori and on the clinical isolate cagA-negative HP61 strain of H. pylori. Tubes containing BJ at concentrations corresponding to $0.5,1,2,4$ and $8 \times$ MIC were inoculated with a suspension of each test strain, yielding to a final bacterial density of $5 \times 10^{6} \mathrm{cfu} / \mathrm{ml}$ and then incubated at $37{ }^{\circ} \mathrm{C}$ under microaerophilic conditions (GENbag microaer for microaerophilic bacteria, BioMerieux). A growth control was also performed. Samples for viable counting were withdrawn at $0,2,4,6,8$, and $24 \mathrm{~h}$. At least four dilutions of each sample were spread in triplicate on Columbia agar plates containing horse blood (7\%, Oxoid), incubated at $37{ }^{\circ} \mathrm{C}$ under microaerohpilic conditions and counted after 3 days.

Time-kill studies were also performed using a combination of each antibiotic and BJ: solutions of the two drugs were added to tubes containing a suspension of single test strains giving a final bacterial concentration of $5 \times 10^{6} \mathrm{cfu} / \mathrm{ml}$. The final concentrations of drugs in each tube were the following: (1) control (without drug); (2) BJ (1 x MIC); (3) AMX (1 x MIC); (4) CLA (1 x MIC); (5) MTZ (1 x MIC); (6) BJ (1 x MIC) and AMX (1 x MIC); (7) BJ (1 x MIC) and CLA (1 x MIC); (8) BJ $(1 \times \mathrm{MIC})$ and MTZ $(1 \times \mathrm{MIC})$. All experiments were performed in triplicate for each strain.

\section{Results}

\section{Minimum inhibitory concentrations}

The MICs of BJ, AMX, CLA and MTZ against the three reference strains and the clinical isolates of $H$. pylori are

Table 1 MICs of bergamot juice (\% v/v) and reference antibiotics (amoxicillin, clarithromycin and metronidazole expressed as $\mu \mathrm{g} / \mathrm{ml}$ ) against ATCC strains and 32 clinical isolates of H. pylori

\begin{tabular}{|c|c|c|c|c|c|}
\hline \multirow[b]{2}{*}{ Compound } & \multicolumn{2}{|l|}{ ATCC } & \multicolumn{3}{|l|}{$\mathrm{Cl}$} \\
\hline & 43504 & 49503 & MIC50 & MIC90 & MIC range \\
\hline$\overline{B J}$ & 2.5 & 5.0 & 2.5 & 5.0 & $0.625-5.0$ \\
\hline AMX & 0.0312 & 0.0156 & 0.0312 & 0.0625 & $0.0156-0.125$ \\
\hline CLA & 0.0625 & 0.0312 & 0.0625 & 0.125 & $0.0312-0.125$ \\
\hline MTZ & 64.0 & 8.0 & 2.0 & 4.0 & $1.0-16.0$ \\
\hline
\end{tabular}

MICs minimal inhibitory concentrations, $B J$ bergamot juice, $A M X$ amoxicillin, $C L A$ clarithromycin, MTZ metronidazole, $\mathrm{Cl}$ clinical isolates of $\mathrm{H}$. pylori 
Table 2 FIC index of the association bergamot juice with reference antibiotics (amoxicillin, clarithromycin and metronidazole) against ATCC strains and 32 clinical isolates of $\mathrm{H}$. pylori

\begin{tabular}{lllllll}
\hline & ATCC & & & $\mathrm{Cl}$ & & \\
\cline { 2 - 3 } \cline { 7 - 8 } Association & 43504 & 49503 & & FICl 50 & FICl 90 & FICl range \\
\hline BJ/AMX & 0.375 & 0.375 & & 0.187 & 0.308 & $0.093-0.49$ \\
BJ/ CLA & 1 & 0.75 & & 0.75 & 1.06 & $0.56-1.24$ \\
BJ/ MTZ & 0.501 & 0.187 & & 0.093 & 0.192 & $0.093-0.75$
\end{tabular}

$B J$ bergamot juice, $A M X$ amoxicillin, CLA clarithromycin, $M T Z$ metronidazole, $C l$ clinical isolates of $H$. pylori

reported in Table 1. Results of negative controls indicated the complete absence of inhibition of all the $H$. pylori strains tested (data not shown). BJ (2.5\%) inhibited the growth of $50 \%$ of the clinical isolates tested, whereas $5 \%(\mathrm{v} / \mathrm{v})$ inhibited $90 \%$. AMX was the most effective antibiotic against the reference strains and the clinical isolates, followed by CLA and MTZ. In the combination assays, the lowest FIC index calculated against the reference strains of $H$. pylori was 0.187 , which was obtained by the combination of BJ with MTZ (Table 2). The same combination produced the lowest FICI 50 and FICI 90 against the 32 clinical isolates of $H$. pylori (Table 2). Although the interpretation of the FIC indices depends on which of the several definitions described in the literature are used, in this study we have considered as synergistic if the FIC index is $</=0.5$, additive or indifferent if $>0.5$ but $</=4$ and antagonistic if $>4$. Synergism was observed between BJ and AMX and between BJ and MTZ against both the reference strains and the clinical isolates of $H$. pylori. Indifference was observed between BJ and CLA for all the combinations tested.

\section{Time-kill curves}

Concentration dependent killing was observed with BJ against all the strains tested (Fig. $1 \mathrm{a}, \mathrm{b}, \mathrm{c}$ and d). At $2 \mathrm{~h}$ there was a $>3 \log 10$ difference in CFU between BJ $(1.25 \%)$ and BJ $(20.0 \%)$ with H. pylori ATCC 49503 (Fig. 1b) and complete bacterial killing was achieved within $8 \mathrm{~h}$ exposure at $\mathrm{BJ}(20 \%$ and $10 \%)$ and within $24 \mathrm{~h}$ exposure at BJ (5\%). BJ gave overall less killing against $H$. pylori ATCC 43504, although a complete bacterial killing was achieved within $6 \mathrm{~h}$ exposure at BJ ( $20 \%$, Fig. 1a). This trend could be explained by the increased resistance to reference antibiotics of $H$. pylori ATCC 43504 compared to $H$. pylori ATCC 49503 (Table 1). The killing curves of $\mathrm{BJ}$ against the clinical isolate HP6 (cagA-positive) and the clinical isolate HP61 (cagA-negative) have shown HP61 was overall more sensitive than HP6 (Fig. 1c and d): a very rapid bactericidal effect was obtained with BJ (20 \%) against HP61 after

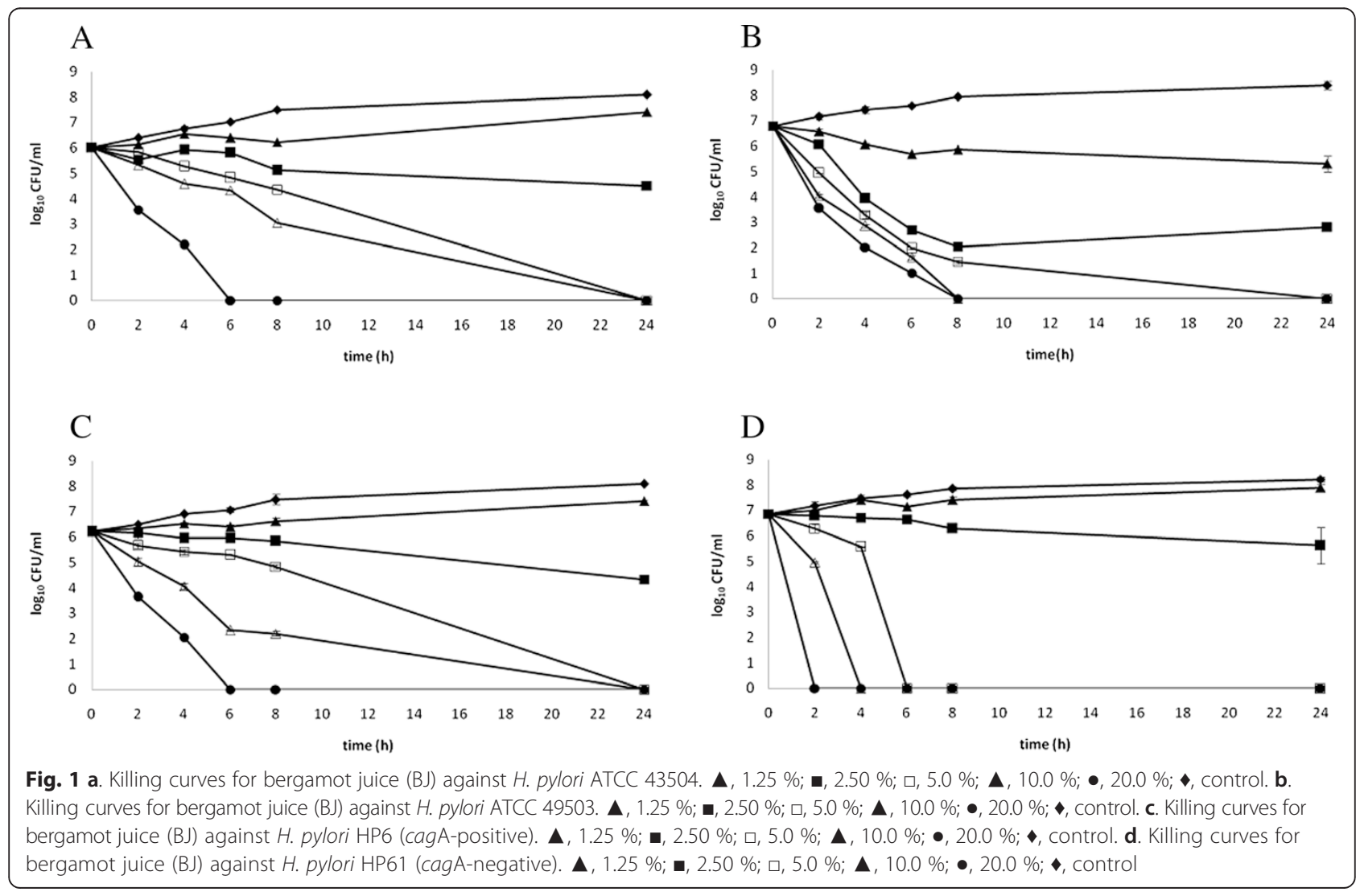


$2 \mathrm{~h}$ exposure, whereas complete bacterial killing was achieved with BJ (20 \%) against HP6 after 6 h exposure. These results suggest the genotype status of the clinical strains of H. pylori may have an impact on their susceptibility to natural antimicrobial compounds.

In the combination assays, the association of $\mathrm{BJ}(1 \mathrm{x}$ MIC) with the reference antibiotics (AMX, 1 x MIC, CLA, $1 \times$ MIC, and MTZ, $1 \times$ MIC) determined a reduction of $>6 \log 10$ for $H$. pylori ATCC 49503 after $8 \mathrm{~h}$ incubation time (Fig. 2b) and for H. pylori ATCC 43504 after $24 \mathrm{~h}$ incubation time (Fig. 2a). Similarly to the killing trends obtained with $\mathrm{BJ}$ alone, the association of $\mathrm{BJ}$ with the three reference antibiotics was more effective against H. pylori ATCC 49503 than H. pylori ATCC 43504. In the association killing curve a synergistic effect was also observed when treating the clinical isolate HP6 (Fig. 2c) and the clinical isolate HP61 (Fig. 2d). The most effective combination was BJ (1 x MIC) and CLA ( $1 \mathrm{x}$ $\mathrm{MIC}$ ) against both clinical isolates, with more rapid bacterial killing achieved with the cagA-positive strain (HP6). This data confirmed the combination between $\mathrm{BJ}$ and the three reference antibiotics had a synergistic effect against H. pylori (both ATCC and clinical isolates). The effect of the association was irrespective of the genotype status of the tested strains.

\section{Discussion}

The results reported in the present study demonstrated for the first time that $\mathrm{BJ}$ was effective against $H$. pylori strains, both alone or in combination with antibiotics. We have recently shown that almond skins were active against the same strains with different virulence irrespective of the cagA and vacA status [14]. In the present study, the genotype status of the clinical isolates affected the suppression of growth exerted by BJ, the cagA-negative strain being more sensitive than the cagA-positive. The three reference antibiotics (AMX, MTZ and CLA) generally used for the treatment of $H$. pylori were effective in combination with BJ: the standardization of combination therapies may be useful against the increasing numbers of multiple drug resistant strains. All $H$. pylori tested in this study were susceptible to amoxicillin ( $\left.\mathrm{MIC}_{90} 0.25 \mu \mathrm{g} / \mathrm{ml}\right)$, two (6\%) out of 32 clinical isolates tested were clarithromycin resistant, one of which isolated from a patient suffering from gastritis harbouring the $c a g \mathrm{~A}+/ \mathrm{vacAs} 1 / \mathrm{m} 1$ genotype, four $(12 \%)$ out of 32 clinical isolates tested were resistant to metronidazole.


Fig. 2 a. Killing curves for bergamot juice (BJ) with reference antibiotics (amoxicillin, AMX, metronidazole, MTZ, clarithromycin, CLA) against $H$,

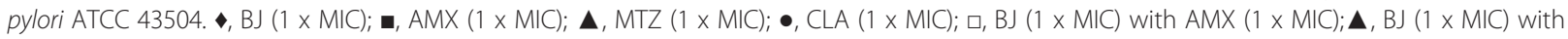
MTZ $(1 \times \mathrm{MIC}) ; \mathrm{O}$, BJ $(1 \times \mathrm{MIC})$ with CLA $(1 \times \mathrm{MIC}) ; \times$, control. b. Killing curves for bergamot juice (BJ) with reference antibiotics (amoxicillin, AMX,

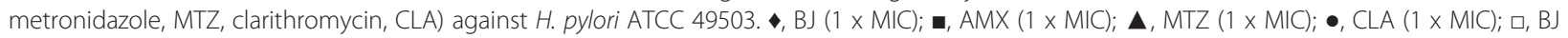

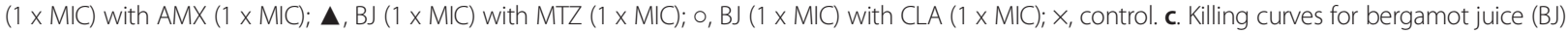
with reference antibiotics (amoxicillin, AMX, metronidazole, MTZ, clarithromycin, CLA) against H. pylori HP6 (cagA-positive). $\bullet$ BJ (1 X MIC); $\mathbf{m}, A M X$ $(1 \times \mathrm{MIC}) ; \mathbf{\Delta}, \mathrm{MTZ}(1 \times \mathrm{MIC}) ; \bullet, \mathrm{CLA}(1 \times \mathrm{MIC}) ; \mathrm{\square}, \mathrm{BJ}(1 \times \mathrm{MIC})$ with $\mathrm{AMX}(1 \times \mathrm{MIC}) ; \boldsymbol{\Delta}, \mathrm{BJ}(1 \times \mathrm{MIC})$ with MTZ $(1 \times \mathrm{MIC}) ; 0, \mathrm{BJ}(1 \times \mathrm{MIC})$ with $\mathrm{CLA}$ $(1 \times M I C) ; \times$, control. d. Killing curves for bergamot juice $(B J)$ with reference antibiotics (amoxicillin, AMX, metronidazole, MTZ, clarithromycin,

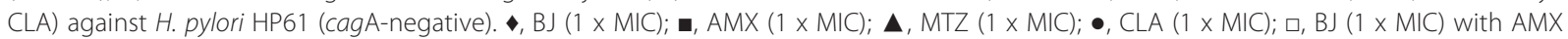
$(1 \times \mathrm{MIC}) ; \mathbf{\Lambda}, \mathrm{BJ}(1 \times \mathrm{MIC})$ with MTZ $(1 \times \mathrm{MIC}) ; 0$, BJ $(1 \times \mathrm{MIC})$ with $\mathrm{CLA}(1 \times \mathrm{MIC}) ; \times$, control 
We have shown that the interactions between $\mathrm{BJ}$ and the reference antibiotics (CLA, MTZ and AMX) can improve the effectiveness of the synthetic antibiotic against H. pylori. Although the exact mechanism of action is not known, we believe that the synergistic effect may be due to the initial damage of the microbial lipid membrane by the plant compound, which would increase the permeability of the bacteria to the antibiotic. Trombetta et al. [28] have demonstrated that the damage of the microbial lipid membrane function by essential oils could be related to the lipid composition and the net surface charge of the membrane, the Gram-negative bacteria presenting a strong negative charge conferred by the lipopolysaccharide. Other reports on the mechanisms of action of isoflavones and chalcones have indicated an inhibition of the urease secreted by $H$. pylori which is crucial for its survival under the acidic conditions of the human stomach $[29,30]$. Several flavone derivatives and other polyphenols in plants are able to inhibit ion and urea conduction and cell vacuolation by VacA, major virulence factor of $H$. pylori [31]. The anti-adhesive properties of BJ could also be responsible for its activity against $H$. pylori $[16,17]$.

The indifferent effect observed by the combination of BJ with CLA against H.pylori could be due to a number of mechanisms, such as inhibition of antibiotic uptake by the bacterial cells compared to MTZ and AMX. Alternatively, direct interaction between the two compounds may result in the reduction of the inhibitory effect. However, in the time-kill studies, the most effective combination was BJ and CLA against both clinical isolates and a synergistic interaction was detected against the two ATCC strains tested.

We have previously demonstrated that polyphenols present in bergamot peel were found to be active against a range of Gram-negative bacteria including Escherichia coli, Pseudomonas putida and Salmonella enterica, and their antimicrobial potency increased after enzymatic deglycosylation [32]. The most representative polyphenols present in bergamot peel were neohesperidin and hesperetin (aglycone), neoeriocitrin and eriodictyol (aglycone), naringin and naringenin (aglycone). We believe that the same compounds present in BJ could be responsible for the antimicrobial effect against $H$. pylori strains [3-7].

\section{Conclusion}

The results of the present study demonstrated that BJ was effective against $H$. pylori strains in vitro, both alone and in combination with antibiotics, and could therefore be used as novel strategy for the treatment of antibiotic resistance. Further studies on the mechanism of action of bergamot juice and its interaction with synthetic antibiotics are warranted.

\section{Abbreviations}

H. pylori: Helicobacter pylori; cagA: Cytotoxin-associated gene; vacA: Vacuolating cytotoxin gene; PAl: Pathogenicity island; PPI: Proton pump inhibitor; AMX: Amoxicillin; CLA: Clarithromycin; MTZ: Metronidazole; BJe: Flavonoid fraction of bergamot juice; MIC: Minimum inhibitory concentration; FIC: Fractional inhibitory concentration.

\section{Competing interests}

The authors declare that they have no competing interests.

\section{Authors' contributions}

AF performed experiments and assisted in drafting the manuscript. CB performed experiments. NF and SC analyzed the data and assisted in data interpretation. GM designed experiments and drafted the manuscript. MN conceived the experiments and edited the manuscript. All authors read and approved the final manuscript.

\section{Acknowledgements}

Research was supported by grants from Sicily Region (PO FESR Sicilia 2007/2013, project "MEPRA", N. 133 of Linea d'Intervento 4.1.1.1, CUP G73F11000050004) and from Calabria Region (PSR Calabria 2007/2013 misura 124, project "ABSIB") to MN.

\section{Author details}

'Dipartimento di Scienze del Farmaco e Prodotti per la Salute, University of Messina, Viale Annunziata, 98168, Messina, Italy. ${ }^{2}$ Dipartimento di Scienze Biologiche e Ambientali, University of Messina, Sal. Sperone 31, 98166 Messina, Italy.

Received: 5 February 2015 Accepted: 7 July 2015

Published online: 30 July 2015

\section{References}

1. Covacci A, Telford JL, Del Giudice G, Parsonnet J, Rappuoli R. Helicobacter pylori virulence and genetic geography. Science. 1999;284(5418):1328-33.

2. Momtaz H, Souod N, Dabiri H. Comparison of the virulence factors of Helicobacter pylori isolated in stomach and saliva in Iran. Am J Med Sci. 2010;340(5):345-9.

3. Hunt RH, Xiao SD, Megraud F, Leon-Barua R, Bazzoli F, van der Merwe S, et al. Helicobacter pylori in developing countries. World Gastroenterology Organisation Global Guideline. J Gastrointestin Liver Dis. 2011;20(3):299-304.

4. Atherton JC, Cao P, Peek Jr RM, Tummuru MK, Blaser MJ, Cover TL. Mosaicism in vacuolating cytotoxin alleles of Helicobacter pylori. Association of specific vacA types with cytotoxin production and peptic ulceration. J Biol Chem. 1995;270(30):17771-7.

5. Akopyants NS, Clifton SW, Kersulyte D, Crabtree JE, Youree BE, Reece CA, et al. Analyses of the cag pathogenicity island of Helicobacter pylori. Mol Microbiol. 1998;28(1):37-53

6. Peek Jr RM, Blaser MJ, Mays DJ, Forsyth MH, Cover TL, Song SY, et al. Helicobacter pylori strain-specific genotypes and modulation of the gastric epithelial cell cycle. Cancer Res. 1999;59(24):6124-31.

7. Rudi J, Kolb C, Maiwald M, Kuck D, Sieg A, Galle PR, et al. Diversity of Helicobacter pylori vacA and cagA genes and relationship to VacA and CagA protein expression, cytotoxin production, and associated diseases. J Clin Microbiol. 1998;36(4):944-8.

8. Mishra S. Is Helicobacter pylori good or bad? Eur J Clin Microbiol Infect Dis 2013;32(3):301-4.

9. Graham DY. Therapy of Helicobacter pylori: current status and issues. Gastroenterology. 2000;118(2 Suppl 1):S2-8.

10. Gerrits MM, van Vliet AH, Kuipers EJ, Kusters JG. Helicobacter pylori and antimicrobial resistance: molecular mechanisms and clinical implications. Lancet Infect Dis. 2006;6(11):699-709.

11. Fukai T, Marumo A, Kaitou K, Kanda T, Terada S, Nomura T. Anti-Helicobacter pylori flavonoids from licorice extract. Life Sci. 2002;71(12):1449-63.

12. Shin JE, Kim JM, Bae EA, Hyun YJ, Kim DH. In vitro inhibitory effect of flavonoids on growth, infection and vacuolation of Helicobacter pylori. Planta Med. 2005;71(3):197-201.

13. Nostro A, Cellini L, Di Bartolomeo S, Di Campli E, Grande R, Cannatelli MA, et al. Antibacterial effect of plant extracts against Helicobacter pylori. Phytother Res. 2005;19(3):198-202. 
14. Bisignano C, Filocamo A, La Camera E, Zummo S, Fera MT, Mandalari G. Antibacterial activities of almond skins on cagA-positive and-negative clinical isolates of Helicobacter pylori. BMC Microbiol. 2013;13:103.

15. Navarra M, Mannucci C, Delbo M, Calapai G. Citrus bergamia essential oil: from basic research to clinical application. Front Pharmacol. 2015;6:36.

16. Delle Monache S, Sanita P, Trapasso E, Ursino MR, Dugo P, Russo M, et al. Mechanisms Underlying the Anti-Tumoral Effects of Citrus bergamia Juice. PLoS One. 2013;8(4):e61484.

17. Navarra M, Ursino MR, Ferlazzo N, Russo M, Schumacher U, Valentiner U. Effect of Citrus bergamia juice on human neuroblastoma cells in vitro and in metastatic xenograft models. Fitoterapia. 2014;95:83-92.

18. Visalli G, Ferlazzo N, Cirmi S, Campiglia P, Gangemi S, Di Pietro A, et al Bergamot Juice Extract Inhibits Proliferation by Inducing Apoptosis in Human Colon Cancer Cells. Anti-Cancer Agent Me. 2014;14(10):1402-13.

19. Ferlazzo N, Visalli G, Smeriglio A, Cirmi S, Lombardo GE, Campiglia P, et al. Flavonoid fraction of orange and bergamot juice protect human lung epithelial cells from hydrogen peroxide-induced oxidative stress. Evid-Based Compl Altern Med. 2015;1: 14

20 Risitano R, Curro M, Cirmi S, Ferlazzo N, Campiglia P, Caccamo D, et al. Flavonoid Fraction of Bergamot Juice Reduces LPS-Induced Inflammatory Response through SIRT1-Mediated NF-kappa B Inhibition in THP-1 Monocytes. PLoS One. 2014;9(9):e107431.0.

21. Impellizzeri D, Bruschetta G, Di Paola R, Ahmad A, Campolo M, Cuzzocrea S, et al. The anti-inflammatory and antioxidant effects of bergamot juice extract (BJe) in an experimental model of inflammatory bowel disease. Clin Nutr. 2014. doi:10.1016/j.clnu.2014.11.012.

22. Marino A, Paterniti I, Cordaro M, Morabito R, Campolo M, Navarra M, et al. Role of natural antioxidants and potential use of bergamot in treating rheumatoid arthritis. Pharma Nutrition. 2015;3(2):53-9.

23. Mollace V, Sacco I, Janda E, Malara C, Ventrice D, Colica C, et al. Hypolipemic and hypoglycaemic activity of bergamot polyphenols: From animal models to human studies. Fitoterapia. 2011;82(3):309-16.

24. Miceli N, Mondello MR, Monforte MT, Sdrafkakis V, Dugo P, Crupi ML, et al. Hypolipidemic effects of Citrus bergamia Risso et Poieteau juice in rats fed a hypercholesterolemic diet. J Agric Food Chem. 2007;55(26):10671-7.

25. Clinical and Laboratory Standards Institute. Performance standards for antimicrobial susceptibility testing; twentieth informational supplement. M100-S22. Wayne: PA: CLSI; 2012.

26. White RL, Burgess DS, Manduru M, Bosso JA. Comparison of three different in vitro methods of detecting synergy: time-kill, checkerboard, and E test. Antimicrob Agents Chemother. 1996:40(8):1914-8.

27. Filocamo A, Bisignano C, D'Arrigo M, Ginestra G, Mandalari G, Galati EM. Norfloxacin and ursolic acid: in vitro association and postantibiotic effect against Staphylococcus aureus. Lett Appl Microbiol. 2011;53(2):193-7.

28. Trombetta D, Castelli F, Sarpietro MG, Venuti V, Cristani M, Daniele C, et al. Mechanisms of antibacterial action of three monoterpenes. Antimicrob Agents Chemother. 2005;49(6):2474-8.

29. Xiao ZP, Shi DH, Li HQ, Zhang LN, Xu C, Zhu HL. Polyphenols based on isoflavones as inhibitors of Helicobacter pylori urease.Bioorgan Med Chem. 2007;15(11):3703-10.

30. Ansari FL, Umbreen S, Hussain L, Makhmoor T, Nawaz SA, Lodhi MA, et al. Syntheses and biological activities of chalcone and 1,5-benzothiazepine derivatives: promising new free-radical scavengers, and esterase, urease, and alpha-glucosidase inhibitors. Chem Biodivers. 2005;2(4):487-96.

31. Tombola F, Campello S, De Luca L, Ruggiero P, Del Giudice G, Papini E, et al. Plant polyphenols inhibit VacA, a toxin secreted by the gastric pathogen Helicobacter pylori. FEBS Lett. 2003;543(1-3):184-9.32.

32. Mandalari G, Bennett RN, Bisignano G, Trombetta D, Saija A, Faulds CB, et al. Antimicrobial activity of flavonoids extracted from bergamot (Citrus bergamia Risso) peel, a byproduct of the essential oil industry. J Appl Microbiol. 2007;103(6):2056-64.

\section{Submit your next manuscript to BioMed Central and take full advantage of:}

- Convenient online submission

- Thorough peer review

- No space constraints or color figure charges

- Immediate publication on acceptance

- Inclusion in PubMed, CAS, Scopus and Google Scholar

- Research which is freely available for redistribution

Submit your manuscript at www.biomedcentral.com/submit 